\title{
MATERIAL DE APOIO AO APRENDIZADO DOS CONCEITOS DE ÁLGEBRA E DE TRIGONOMETRIA ASSOCIADOS ÀS ANÁLISES DE CIRCUITOS ELÉTRICOS EM CORRENTE ALTERNADA
}

DOI: 10.37702/2175-957X.COBENGE.2021.3616

Marcos Roberto Fernandes Filho - marcosrff.2001@gmail.com

Instituto Federal de Santa Catarina

Rua Príncipe 64

88032-325 - Florianopolis - SC

Cícero Eduardo Dick Junior - cicero.edjr@gmail.com

Instituto Federal de Santa Catarina

Rua Rodolfo Weiss 310

88135-218 - Palhoça - SC

Mauro Tavares Peraça - peraca@ifsc.edu.br

Instituto Federal de Santa Catarina

Mauro Ramos 950

88020-300 - Florianópolis - SC

Alexandre Leizor Szczupak - alexandre.leizor@ifsc.edu.br

Instituto Federal de Santa Catarina

Rua Crispim Mira 171

88020-540 - Florianópolis - SC

Resumo: $O$ estudo de circuitos elétricos em corrente alternada é fundamentado em análises matemáticas que envolvem conceitos de álgebra e de trigonometria. Apesar disso, a compreensão desses conceitos por estudantes dos cursos de Engenharia Elétrica e Engenharia Eletrônica não é sempre sólida. Comumente, os estudantes apresentam dificuldades para interpretar e relacionar conceitos matemáticos à análise de circuitos. Propomos, para mitigar esse problema, o uso de um conjunto de aplicativos computacionais desenvolvidos especificamente para facilitar a conexão de conceitos de álgebra e de trigonometria à análise de circuitos em corrente alternada. Cada aplicativo aborda um aspecto distinto do estudo desses circuitos de modo a possibilitar ao estudante um maior controle sobre o ritmo de seu estudo. O material, desenvolvido e disponibilizado através da 


\section{COBENCE Evento online

plataforma GeoGebra, também pode ser utilizado por docentes durante suas aulas para tornar as atividades mais dinâmicas.

Palavras-chave: Circuitos elétricos. Álgebra. Trigonometria. GeoGebra. 


\section{MATERIAL DE APOIO AO APRENDIZADO DOS CONCEITOS DE ÁLGEBRA E DE TRIGONOMETRIA ASSOCIADOS ÀS ANÁLISES DE CIRCUITOS ELÉTRICOS EM CORRENTE ALTERNADA}

\section{INTRODUÇÃO}

A teoria dos circuitos elétricos voltados para o processamento de sinais senoidais em regime estacionário é fundamentada em conceitos matemáticos de álgebra, de trigonometria e de aritmética com números complexos (BOYLESTAD, 2012, cap. 13-19; GUSSOW, 2008, cap 11-14). A compreensão desses conceitos por estudantes das áreas de Engenharia Elétrica e de Engenharia Eletrônica Ihes permite perceber as conexões entre a teoria matemática e a manifestação física dos sinais elétricos senoidais. Infelizmente, essa compreensão não é sólida entre todos os estudantes (MORALES; PUHL; LIMA, 2013; PUHL; MÜLLER; LIMA, 2019; OLIVEIRA et al., 2020). Muitos apresentam dificuldades para associar os conceitos matemáticos fundamentais da área às suas aplicações práticas.

Para ajudar a mitigar esse problema, desenvolvemos uma biblioteca de aplicativos computacionais que pode ser usada como ferramenta de suporte ao entendimento das conexões entre a matemática e a física dos circuitos em corrente alternada (como são chamados, comumente, os circuitos voltados para o processamento elétrico de sinais senoidais em regime estacionário). Os aplicativos da biblioteca possuem interfaces gráficas interativas e estão disponíveis online, onde podem ser acessados livremente através de navegadores de internet.

O material, desenvolvido e disponibilizado através da plataforma GeoGebra (GEOGEBRA, 2021), pode ser utilizado por docentes como material de apoio às suas aulas. Entre as vantagens do seu uso está a agilidade para a construção e a alteração das propriedades de figuras trigonométricas, desenhos de formas de ondas e representações fasoriais. Os aplicativos podem ser usados em diversas disciplinas de cursos de graduação em Engenharia Elétrica e Engenharia Eletrônica, bem como em cursos de nível técnico.

O uso do software GeoGebra em aplicativos voltados para o estudo de circuitos elétricos em corrente alternada foi realizado por Stump e Abar $(2013,2014)$ em estudos voltados aos cursos de Engenharia Elétrica. De acordo com Stump e Abar (2014, p.44), "Inovação tecnológica na prática docente não se caracteriza por transferir aulas para o computador, mas sim utilizar os recursos disponíveis como simulações computacionais, animações e recursos interativos como complemento ao ensino e aprendizagem." Tendo este conceito em mente, não buscamos realizar uma transferência de conteúdo entre plataformas, mas criar um conjunto de ferramentas que aproveita as possibilidades de interação que um aplicativo computacional implementado na plataforma GeoGebra pode fornecer.

\section{$2 \quad$ APLICATIVOS DESENVOLVIDOS}

Cada aplicativo desenvolvido aborda um aspecto distinto do estudo de circuitos em corrente alternada. Desta forma, o estudante não é sobrecarregado com muitas informações em um único aplicativo e pode estudar o material de forma paulatina, de acordo com o seu próprio tempo de estudo. De forma similar, o docente que utilizar os 
aplicativos como material de apoio, pode incorporá-los às suas atividades de forma modular, sem precisar mostrar conteúdos diversos simultaneamente.

Os aplicativos desenvolvidos permitem grande interação. O usuário pode alterar os parâmetros de acordo com seu estudo específico, além de utilizar recursos como comandos deslizantes e animações para visualizar o comportamento das ondas de tensão e de corrente. Deste modo, obtém-se maior agilidade na construção e alteração das propriedades de figuras trigonométricas, desenhos de ondas e representações fasoriais de sinais. A agilidade na criação de construções gráficas propiciada pelo uso do GeoGebra foi observada por De Mamam (2017, p.7) que apontou, como uma das consequências positivas, a disponibilidade de mais tempo para discussões conceituais em sala de aula.

Apesar desse tipo de construção gráfica, essencial ao estudo de circuitos em corrente alternada, usualmente demandar muito tempo para ser realizada manualmente, não é nossa proposta substituí-la sempre pelo uso dos aplicativos. Esse tipo de atividade manual traz importantes contribuições ao aprendizado, apesar de facilmente se tornar morosa para docentes e discentes. Acreditamos que os aplicativos são melhor utilizados se encarados como material complementar, usados para melhorar a dinâmica dos estudos.

\subsection{Aplicativo Senoides}

Este aplicativo serve ao estudo dos conceitos de amplitude, frequência e ângulo de fase de um sinal senoidal. Podemos alterar esses parâmetros através de controles deslizantes, observando simultaneamente as variações resultantes na forma de onda mostrada. Há também três botões disponíveis com as finalidades: "Animar", "Reiniciar Onda" e "Alternar Onda". O botão "Animar" é responsável pela variação automática da forma de onda de acordo com os valores mínimo e máximo de um de seus parâmetros. $O$ desenho é atualizado continuamente conforme o valor do parâmetro escolhido oscila entre seus valores mínimo e máximo. O botão "Reiniciar Onda" serve para restaurar a onda e seus parâmetros iniciais, a configuração inicial. O botão "Alternar Onda" permite que o usuário alterne a forma de onda entre a opção de tensão ou a de corrente, sendo a onda de tensão representada em vermelho e a onda de corrente representada em azul, como mostrado na Figura 1. 
Figura 1 - Aplicativo Senoides

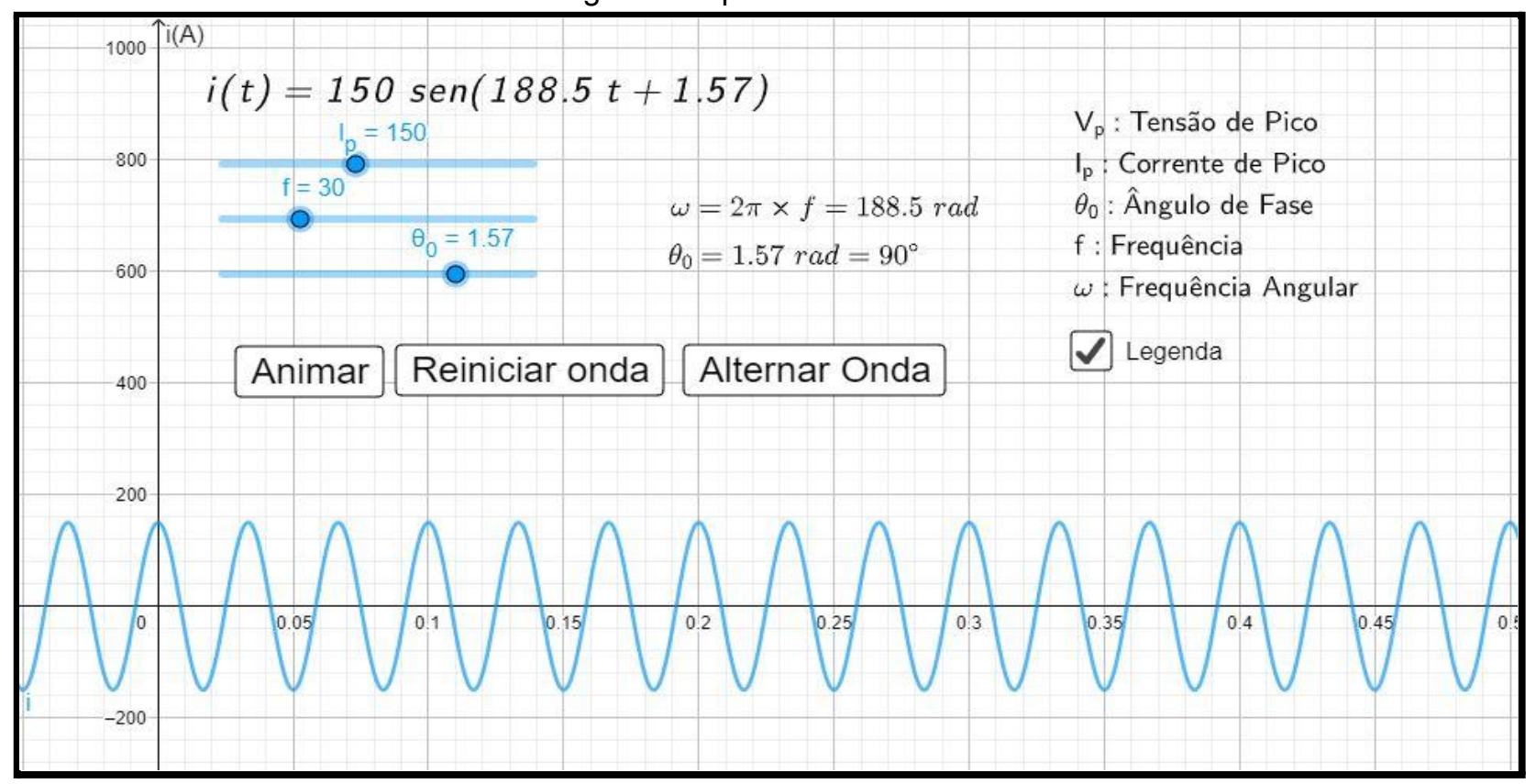

Fonte: Autoria própria.

\subsection{Aplicativo Senoides e Fasores}

O aplicativo Senoides e Fasores serve ao estudo das relações entre os parâmetros de uma senoide e a sua representação fasorial, tanto na forma gráfica quanto nas suas representações retangular e polar. A variação dos controles deslizantes permite a alteração dos valores de amplitude, frequência e ângulo de fase. Os resultados dessas alterações são mostrados de forma imediata. Do lado esquerdo observa-se a onda construída a partir dos valores indicados e do lado direito as consequências que essas modificações têm na representação fasorial.

Figura 2 - Aplicativo Senoides e Fasores.

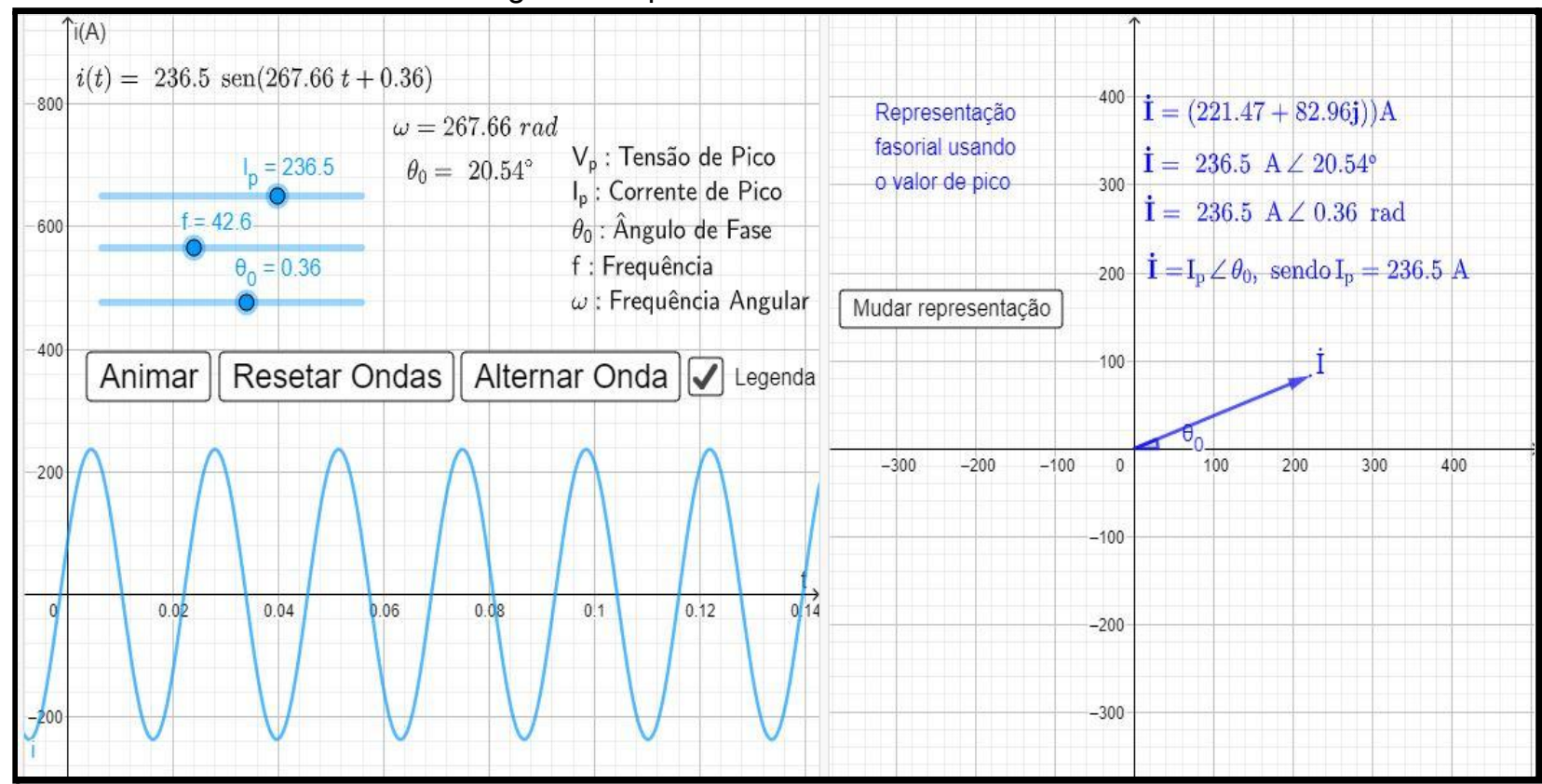

Fonte: Autoria própria.

\subsection{Aplicativo Soma de Senoides e Fasores}


Este aplicativo permite ao usuário visualizar a representação senoidal e fasorial para a soma de duas ondas que têm a mesma frequência porém têm amplitudes e ângulos de fase independentes. O usuário pode alterar os valores dos parâmetros das ondas senoidais através de controles deslizantes. As formas de onda são apresentadas do lado esquerdo da tela, junto com o resultados das equações de amplitude, frequência angular e ângulo de fase. Do lado direito é possível visualizar tanto a representação fasorial das ondas independentes, quanto a representação fasorial da onda resultante. $O$ uso de cores diferentes associadas aos parâmetros e gráficos de cada onda, auxilia na identificação de cada elemento formador da construção.

Figura 3 - Aplicativo Soma de Senoides e Fasores.

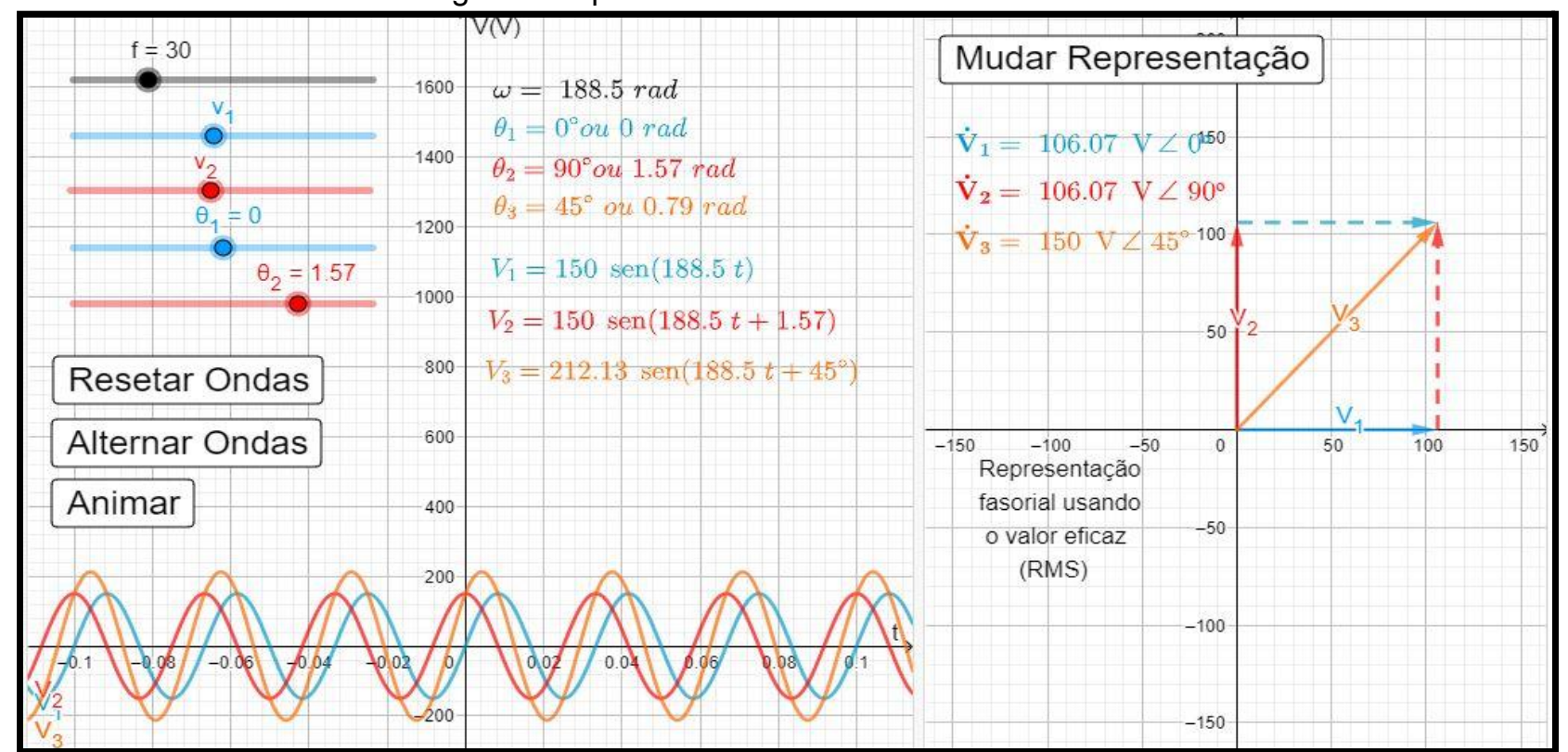

Fonte: Autoria própria.

\subsection{Aplicativo Associação de Impedâncias}

Este aplicativo é voltado para o estudo trigonométrico da associação de impedâncias em circuitos em corrente alternada. O usuário pode interagir com seis circuitos diferentes: RL Série, RC Série, RLC Série, RL Paralelo, RC Paralelo e RLC Paralelo. Os circuitos podem ser alternados a partir do botão presente acima de sua própria figura na parte superior esquerda do aplicativo. É possível inserir a frequência em que a fonte opera e também os valores de três componentes: resistor (R), capacitor (C) e indutor (L). O valor numérico da impedância resultante é mostrado, na forma retangular e na forma polar, do lado esquerdo da interface. O lado direito da interface mostra a construção trigonométrica dessa impedância.

Figura 4 - Aplicativo Associação de Impedâncias. 


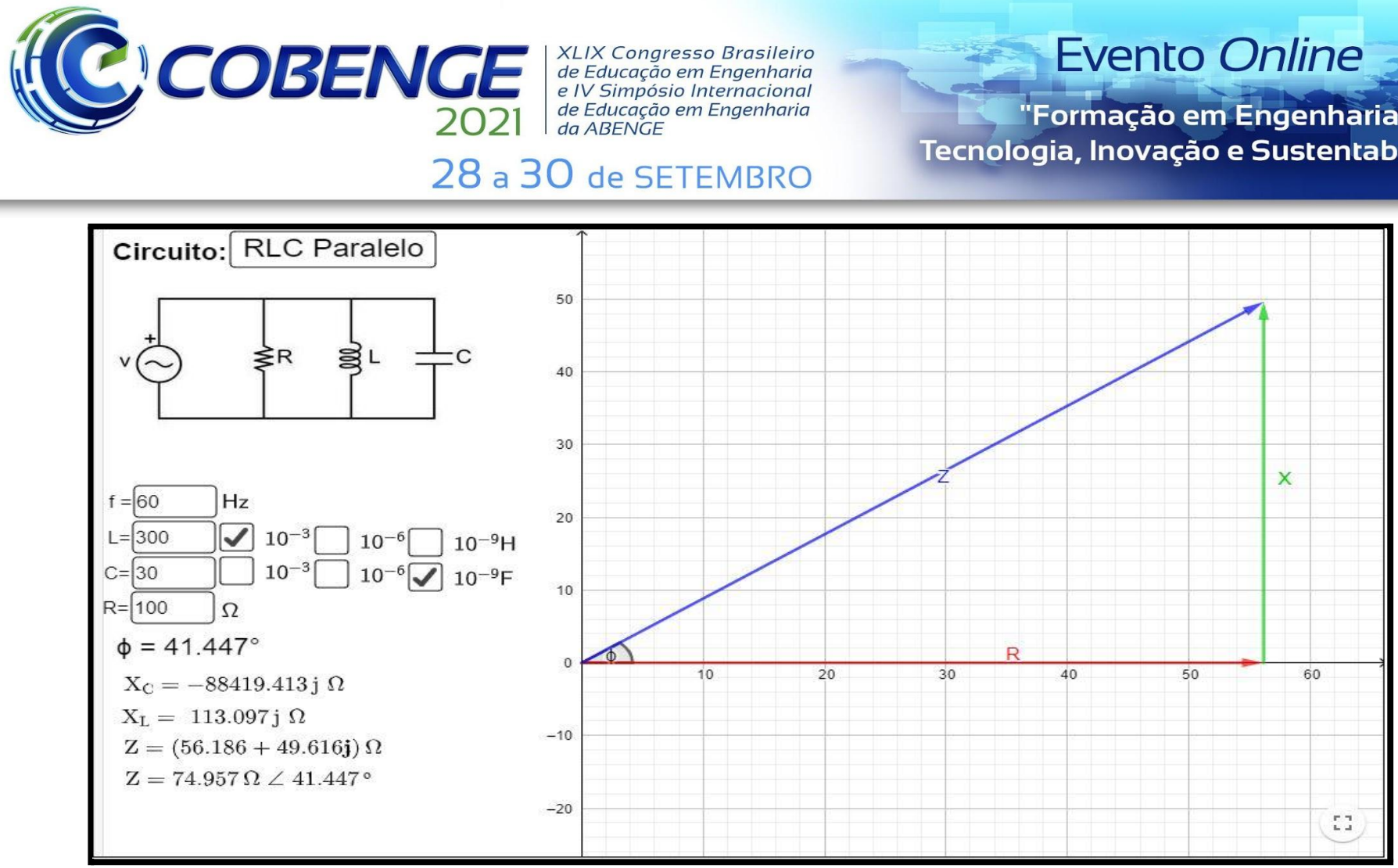

Fonte: Autoria própria.

\subsection{Aplicativo Triângulo de Impedâncias}

O aplicativo Triângulo de Impedâncias apresenta uma forma alternativa para o estudo da associação de impedâncias. Neste aplicativo, o usuário insere diretamente os valores de resistência e de reatância, não se limitando assim a um circuito específico como no aplicativo anterior, Associação de Impedâncias. Os resultados numéricos são apresentados logo abaixo dos valores introduzidos pelo usuário, sendo possível observar na parte esquerda da interface a representação vetorial do triângulo de impedâncias.

Figura 5 - Aplicativo Triângulo de Impedâncias

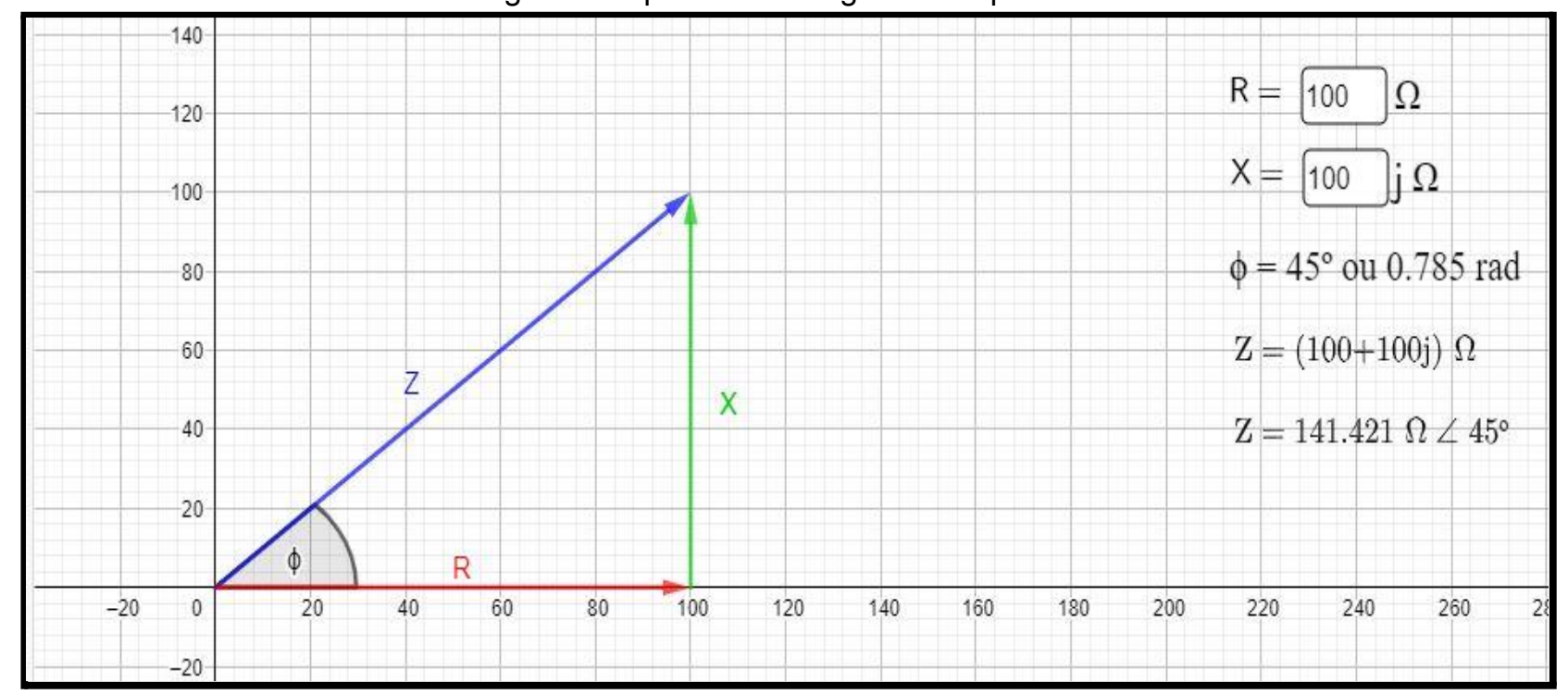

Fonte: Autoria própria.

\subsection{Aplicativo Triângulo de Potências}

A função deste aplicativo é auxiliar o estudo das relações entre as potências ativa, reativa e aparente, através do triângulo retângulo. Na parte esquerda do aplicativo pode-se inserir os dados da tensão eficaz, da corrente eficaz e do ângulo de fase. Logo abaixo dos valores introduzidos é possível observar, respectivamente, os resultados do 
fator de potência $(F P)$, da potência aparente $(S)$, da potência ativa $(P)$ e da potência reativa (Q). A partir dos valores obtidos constrói-se imediatamente, ao lado direito da interface, a representação vetorial de um triângulo de potências.

Figura 6 - Aplicativo Triângulo de Potência

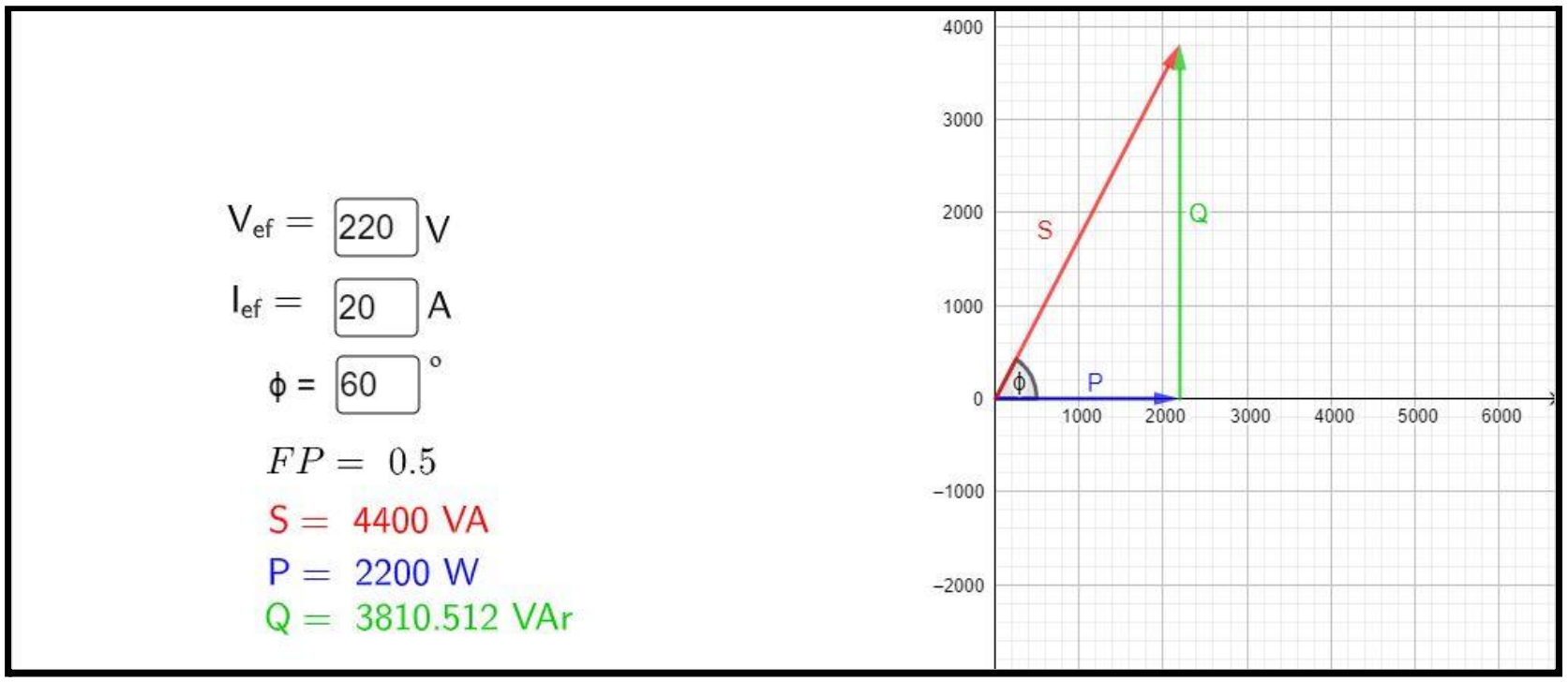

Fonte: Autoria própria.

\subsection{Aplicativo Tensão, Corrente e Potência Instantânea}

Este aplicativo permite ao usuário visualizar a potência média e a forma de onda da potência instantânea referentes a diferentes combinações entre ondas de tensão e de corrente. Os controles deslizantes são responsáveis por variar a amplitude, a frequência e o ângulo de fase das ondas de tensão e de corrente. Ao lado dos controles deslizantes são disponibilizados os resultados das equações das ondas e as conversões de seus ângulos de fase de radianos para graus. Na parte inferior do aplicativo aparece a representação da onda de potência instantânea, que é modificada em tempo real de acordo com as alterações feitas pelo usuário nos valores dos parâmetros das ondas de tensão e de corrente. 


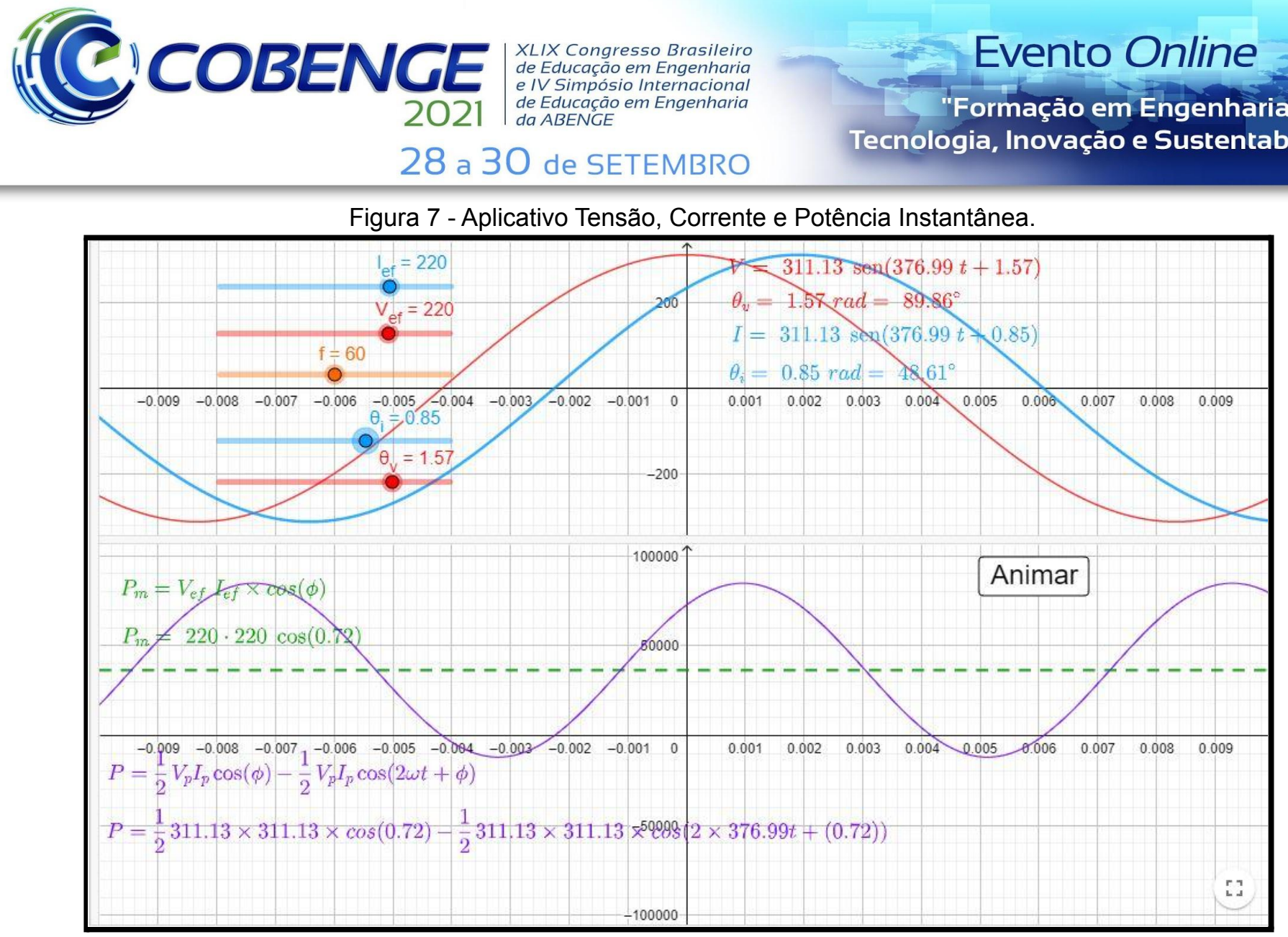

Fonte: Autoria própria.

\section{ACESSO AOS APLICATIVOS}

Todos os aplicativos estão disponíveis na internet em um sítio GeoGebra (ANÁLISE, 2021). O acesso aos aplicativos é livre e seus códigos estão sob uma licença Creative Commons CC-BY-SA 3.0 (CREATIVE COMMONS, 2021).

\section{CONSIDERAÇÕES FINAIS}

A percepção das conexões entre a teoria matemática e a manifestação física dos sinais elétricos senoidais é um aspecto importante da formação de engenheiros eletricistas e eletrônicos, bem como na formação de tecnólogos e técnicos nessas áreas. A biblioteca de aplicativos apresentada pode servir como um caminho facilitador dessa percepção.

A biblioteca está disponível online para que os estudantes possam acessá-la de onde estiverem e possam usá-la tanto como ferramenta sistemática de estudo, quanto como material de consulta eventual. No momento da construção deste texto, o mundo passa por uma pandemia que causou grandes prejuízos à educação. Aplicativos computacionais voltados para a educação, como os apresentados neste texto, podem servir para amenizar os impactos do isolamento social na formação de estudantes.

A biblioteca também pode ser utilizada por docentes em sala de aula. Seus recursos visuais oferecem praticidade e dinâmica ao docente, reduzindo o tempo que esse precisa usar na construção de representações gráficas. Assim, seu foco pode voltar-se mais facilmente para discussões de conteúdo, aproveitando melhor o tempo limitado das aulas. 


\section{Agradecimentos}

Agradecemos o apoio dado pelo Programa de Integração da Pesquisa e Extensão ao Ensino do Instituto Federal de Santa Catarina, Câmpus Florianópolis, através da segunda chamada do Edital 18/2019/PROEX/PROPPI.

\section{REFERÊNCIAS}

ANÁLISE de circuitos em corrente alternada: aplicativos computacionais, 2021. Disponível em: https://www.geogebra.org/u/analise-ca-fln. Acesso em: 08 mai. 2021.

BOYLESTAD, Robert L. Introdução à análise de circuitos. 12. ed. São Paulo: Pearson, 2012.

CREATIVE COMMONS. Attribution-ShareAlike 3.0 Unported, Disponível em: https://creativecommons.org/licenses/by-sa/3.0/. Acesso em: 08 mai. 2021.

DE MAMAM, Andréia S.; QUARTIERI, M. T.; NEIDE, Italo G. Tecnologias no ensino: experiência em uma disciplina introdutória nos cursos de engenharia. XLV Congresso Brasileiro de Educação em Engenharia, Joinville, 2017.

GEOGEBRA: software de matemática dinâmica. Versão 6.0.640.0, 2021. Disponível em: http://www.geogebra.org/ Acesso em: 08 mai. 2021.

GUSSOW, Milton. Eletricidade Básica. 2. ed. São Paulo: Bookman, 2008.

MORALES, Andréa C.; PUHL, Cassiano S.; LIMA, Isolda G. de. Números Complexos e Corrente Alternada: Um Contexto Interdisciplinar. In: XLI Congresso Brasileiro de Educação em Engenharia, 2013, Gramado. Anais. Gramado.

OLIVEIRA, Lucas D.; RAMOS, Tiago C.; CARNEIRO, Joaquim A. S. A. O.; LANDI JÚNIOR, Salmon. Conhecimentos de Matemática básica de graduandos nos anos iniciais de Engenharia: desafios, fragilidades e enfrentamentos possíveis. Boletim online de Educação Matemática, Florianópolis, v.8, n.16, p. 134-152, 2020.

PUHL, Cassiano S.; MÜLLER, Thaísa J.; LIMA, Isolda G. de. Lacunas de aprendizagem de números complexos: um estudo de caso com acadêmicos de engenharia. In: XLVII Congresso Brasileiro de Educação em Engenharia, 2019, Fortaleza. Anais. Fortaleza.

STUMP, Sandra M. D.; ABAR, Celina A. A. P. Objetos de aprendizagem para ensino de circuitos elétricos em regime estacionário com o uso de números complexos em um curso de engenharia elétrica. VIII International Conference on Engineering and Computer Education, ICECE 2013, Luanda, Angola, p. 333-337, 2013.

STUMP, Sandra M. D.; ABAR, Celina A. A. P. Possíveis contribuições da utilização de objetos de aprendizagem para a compreensão de circuitos elétricos em um curso de engenharia elétrica. CADERNO DE FísICA DA UEFS , Feira de Santana, 2014. 


\title{
SUPPORT MATERIAL FOR THE STUDY OF ALGEBRAIC AND TRIGONOMETRIC CONCEPTS ASSOCIATED WITH THE ANALYSIS OF STEADY-STATE ALTERNATING CURRENT IN ELECTRIC CIRCUITS
}

\begin{abstract}
The study of steady-state alternating current in electric circuits is based on mathematical analysis that comprehends algebraic and trigonometric concepts. Despite that, the comprehension of those concepts by Electrical and Electronic engineering students isn't always solid. Commonly, they have difficulties to interpret and to relate those mathematical concepts to circuit analysis. We propose the use of a collection of applets to mitigate this problem. These applets were created specifically to facilitate the connection of algebraic and trigonometric concepts to the analysis of electric circuits under steady-state alternating current. Each applet deals with a distinct aspect of the analysis, so that students can have more control over the pace of their studies. The material, developed and made available through the GeoGebra platform, can also be used by teachers to make classes activities more dynamic.
\end{abstract}

Keywords: Electric circuits. Algebra. Trigonometry. GeoGebra. 